\title{
Cervical Spine MR Imaging Findings of Patients with Hirayama Disease in North America: A Multisite Study
}

\author{
V.T. Lehman, P.H. Luetmer, E.J. Sorenson, R.E. Carter, V. Gupta, G.P. Fletcher, L.S. Hu, and A.L. Kotsenas
}

\begin{abstract}
BACKGROUND AND PURPOSE: Most studies of HD have been conducted in Asia, particularly Japan. To characterize the MR imaging findings of North American patients with HD, we reviewed neutral and flexion cervical MR imaging examinations performed for possible HD at 3 academic medical centers located in the Southeastern, Southwestern, and Midwestern regions of the United States.
\end{abstract}

MATERIALS AND METHODS: Three neuroradiologists assessed the MR imaging examinations in a blinded fashion and reached a consensus rating for LOA of the posterior dura to the spine, lower spinal cord atrophy, spinal cord T2 hyperintensity, loss of cervical lordosis, anterior dural shift with flexion, and confidence of imaging diagnosis. Final reference diagnosis was established separately with a retrospective chart review by a neurologist.

RESULTS: Twenty-one patients met the criteria for HD, all were North American males and all who reported their race were white. Seventeen patients did not meet the criteria and served as controls. Four imaging attributes, LOA, dural shift with flexion, consensus diagnosis of neutral images, and consensus diagnosis of combined neutral and flexion images were all able to discriminate the group with HD from the group without HD ( $P<.05$ for each). Findings of HD were often present on neutral images, but the addition of flexion images increased diagnostic confidence.

CONCLUSIONS: MR imaging findings in white North American patients with HD include LOA on neutral images and forward displacement of the dura with flexion. Findings are often present on neutral MR images and, in the appropriate clinical scenario, should prompt flexion MR imaging to evaluate anterior dural shift.

ABBREVIATIONS: EMG = electromyography; $\mathrm{HD}=$ Hirayama Disease; $L \mathrm{LOA}=$ loss of attachment

$\mathbf{H}^{\mathrm{D}}$ $\mathrm{D}$ is a rare condition characterized by asymmetric muscle weakness and atrophy in the C8-T1 distribution. ${ }^{1}$ Classically, adolescent males develop progressive weakness that stabilizes after several years. The pathomechanism is debated, but the prevailing theory postulates that insufficient growth of the dura relative to the spinal column during puberty allows forward dis-

Received February 15, 2012; accepted after revision April 16.

From the Division of Neuroradiology (V.T.L., P.H.L., A.L.K.), Department of Radiology, and Department of Neurology (E.J.S.), Mayo Clinic College of Graduate Medical Education, Rochester, Minnesota; Division of Biomedical Statistics and Informatics (R.E.C.), Mayo Clinic Department of Health Sciences Research, Rochester, Minnesota; Division of Neuroradiology (V.G.), Department of Radiology, Mayo Clinic College of Graduate Medical Education, Jacksonville, Florida; and Division of Neuroradiology (G.P.F., L.S.H.), Department of Radiology, Mayo Clinic College of Graduate Medical Education, Scottsdale, Arizona.

Paper previously presented at: Annual Meeting of the American Society of Neuroradiology and The Foundation of the ASNR Symposium, April 21-26, 2012; New York, New York.

Please address correspondence to Vance T, Lehman, MD, Department of Radiology, 200 First St SW, Rochester, MN 55905; e-mail: lehman.vance@mayo.edu

http://dx.doi.org/10.3174/ajnr.A3277 placement of the dura in flexion with compression of the spinal cord, possibly leading to ischemia of the anterior horn cells at C8 and $\mathrm{T} 1 .^{2}$ Most reported cases have been from Asia, particularly Japan, ${ }^{1}$ though some have been reported from India, the Middle East, and Europe. ${ }^{3-6}$ To date, there have only been a handful of cases reported from North America, and to our knowledge, there has been no published study characterizing the MR imaging features of this disease in North American patients. ${ }^{7-11}$

MR imaging findings reported in patients from Southeast Asia and Japan include LOA of the dura to the lamina, asymmetric lower cervical spinal cord atrophy, spinal cord T2 hyperintensity, loss of cervical lordosis in the neutral position, and forward displacement of the dura with flexion MR imaging. ${ }^{8}$

In our experience, HD may be more common in North America than the current literature indicates. During the past several years, we have observed an increase in the number of requests for flexion cervical MR imaging for the evaluation of possible HD. To characterize the MR imaging findings of North American patients with $\mathrm{HD}$, we reviewed neutral and flexion MR images of patients 
with possible HD obtained between 1995 and 2011 at 3 academic medical centers located in the Southeastern, Southwestern, and Midwestern regions of the United States.

\section{MATERIALS AND METHODS \\ Patient Selection}

Institutional review board approval for this retrospective Health Insurance Portability and Accountability Act-compliant study was obtained, and informed consent was waived. We searched our radiology information system and electronic medical record data bases by using the keywords "Hirayama" and "flexion MR." We reviewed the charts and images of all patients who had been referred for a flexion cervical MR imaging examination for consideration of HD between January 1, 1995, and June 9, 2011. Patients referred for a flexion MR imaging examination with an alternate indication such as trauma were excluded. Age, sex, and ethnicity did not affect inclusion.

\section{Image Review}

All studies were performed at 1.5T. Because the radiographic examinations were performed during an extended time, on several different MR imaging scanners, and at several different institutions, there was variability in the MR images obtained. Most patients had a neutral sagittal T1-weighted series, a neutral sagittal T2-weighted series, a neutral axial fast spin-echo T2-weighted series and/or neutral axial T2-weighted gradient-echo series, and a flexion sagittal T2- or T1-weighted series.

Each MR imaging examination was reviewed by 3 neuroradiologists (P.H.L., A.L.K., V.T.L.) with 21, 12, and 1 year of experience who were blinded to clinical data and final diagnosis. If a patient had numerous MR imaging examinations with cervical flexion, only the most recent was scored to establish a consistent method. All findings were reached by consensus. The neutral images were evaluated first, without knowledge of the findings of the flexion series.

On neutral studies, cervical lordosis was scored as either normal or abnormal and LOA was determined as either present or absent at each cervical level and the first thoracic level by using the methods described by Chen et al. ${ }^{8}$ Cervical lordosis was considered normal when the $\mathrm{C} 3-\mathrm{C} 6$ vertebral bodies were all anterior to a line drawn from the posterior cortex of $\mathrm{C} 2$ to the posterior cortex of C7. LOA was considered present when the thecal sac demonstrated separation from the lamina over a distance of greater than one-third of an adjacent lamina. This finding at a single level on 1 side was considered sufficient to score the presence of LOA for the examination. Both axial T2 images and axial gradient-echo images were used in combination to evaluate LOA.

Asymmetric spinal cord flattening at the C5-T1 levels, defined as asymmetric loss of normal oval spinal cord configuration, was determined by using a combination of neutral sagittal and axial images. Confirmation on axial images was mandatory. If neutral images were not available, this was assessed on sagittal and axial flexion images. Asymmetric spinal cord flattening was recorded as either present or absent, and the level of abnormality was noted. Spinal cord flattening with a corresponding disk herniation was not scored.

Spinal cord T2 hyperintensity at the C5-T1 levels was deter- mined by using neutral sagittal and axial T2-weighted images. If neutral images were not available, this was assessed on sagittal and axial flexion images. T2 hyperintensity was recorded as either present or absent. T2 hyperintensity was not scored if there was a corresponding disk herniation, if the signal was thought to represent the central canal of the spinal cord, or if it was limited to the posterior spinal cord.

Before viewing the flexion MR imaging series, the reviewers rated each study as not $\mathrm{HD}$, possible $\mathrm{HD}$, or $\mathrm{HD}$ on the basis of the neutral MR imaging series findings alone. This rating represented the readers' overall subjective impression and degree of confidence rather than a quantitative measure because multiple findings contribute to a diagnostic conclusion in clinical practice. Flexion studies were evaluated primarily for anterior shift of the dura. The degree of displacement was recorded in millimeters on sagittal images. The precise degree of flexion could not be controlled, given the retrospective study design. The reviewers then rated each study for overall diagnostic impression, including neutral and flexion images, as not $\mathrm{HD}$, possible $\mathrm{HD}$, or $\mathrm{HD}$.

\section{EMG Analysis}

A board-certified neurologist (E.J.S.), fellowship-trained in EMG, with 16 additional years' experience in EMG study interpretation evaluated the EMG studies from all patients without any additional clinical or imaging information. For patients with multiple EMG examinations, the EMG that corresponded most closely in time to the reviewed MR image was used. Each left and right nerve C5-T1 was scored independently for involvement or no involvement. The chronicity of EMG findings was recorded as acute, subacute, or chronic. The severity was recorded as mild, moderate, severe, or very severe (1-4, respectively). The myotomal level with the greatest degree of abnormality was recorded.

\section{Chart Review}

A board-certified neurologist (E.J.S.) reviewed the clinical notes of all included patients after the EMG data had been collected, to determine the final clinical diagnosis. Established clinical criteria were used for a diagnosis of HD: 1) symptom onset in late adolescence or young adulthood, 2) motor-only syndrome, 3) motor involvement restricted to the upper extremities, 4) asymmetric muscle changes, 5) a period of clinical progression followed by relative stability, and 6) no alternative explanation for symptoms identified during the evaluation. ${ }^{1}$ This clinical diagnosis served as our criterion standard. Although there are reports of bilaterally symmetric HD and of patients with slight hypoesthesia in the hands, these cases are exceptions and we maintained the classic criteria. ${ }^{12}$ We did not assign a specific diagnosis to patients who did not fulfill the criteria for HD, but the final diagnoses provided in the charts given by the patients' neurologists were recorded.

Demographic features at the time of the MR imaging examination, including age, sex, self-reported ethnicity, and country of residence, were documented.

\section{Data Analysis}

We determined the sensitivity and specificity of the radiologists' predictions of diagnosis on the basis of neutral and flexion MR images. The diagnostic performance of the specific radiographic 
Table 1: Demographic characteristics of patients meeting clinical criteria for Hirayama disease compared with those not meeting clinical criteria

\begin{tabular}{lcc}
\hline & $\begin{array}{c}\text { Patients with } \\
\text { Hirayama Disease } \\
(\mathbf{n}=\mathbf{2 1})\end{array}$ & $\begin{array}{c}\text { Patients without } \\
\text { Hirayama Disease } \\
(\mathbf{n}=17)\end{array}$ \\
\hline Age in years $^{\text {a }}$ (mean) (range) & $24(17-61)$ & $41(15-68)$ \\
Male sex (No.) (\%) & $21(100.0)$ & $11(64.7)$ \\
Residence in North & $21(100.0)$ & $16(94.1)$ \\
America, (No.) (\%) & & \\
White (No.) $\%)$ & $20(95.2)$ & $14(82.3)$ \\
Asian (No.) (\%) & $0(0)$ & $1(5.9)$ \\
Other or unknown race & $1(4.7)$ & $2(11.8)$ \\
\hline
\end{tabular}

${ }^{a}$ Age determined at time of clinical evaluation.

findings of abnormal spinal curvature, LOA, anterior dural shift, spinal cord T2 hyperintensity, asymmetric spinal cord flattening, and the presence of the greatest EMG involvement at the C8 level with severe-to-very-severe changes were also assessed. Score confidence intervals for sensitivity and specificity were computed to account for sampling variation in the data. To summarize the diagnostic performance of the MR imaging attributes or findings, we calculated the odds ratio and 95\% large-sample confidence intervals. For instances when there was a zero cell (eg, 100\% specificity), the empiric logit estimator was used. This estimator added 0.5 to each cell count in the contingency table before calculating the odds ratio. ${ }^{13}$ A 2-tailed Fisher exact test was used to test for significant discrimination between patients with HD and controls with respect to the MR imaging attributes and findings. $P$ values $<.05$ were considered significant. Statistical analysis was conducted by using the SAS System, Version 9.3 (SAS Institute, Cary, North Carolina).

\section{RESULTS}

\section{Initial Search and Chart Review}

Forty-two patients met initial search criteria and were evaluated. Two patients were excluded because the flexion MR image was an outside study in hard copy format only and was no longer archived for review. Two patients were excluded because they could not be confidently classified clinically into either a diagnosis of HD or not HD due to inadequate historical documentation, leaving 38 included patients for this study. Demographics of these patients are outlined in Table 1.

Twenty-one (55\%) patients met clinical criteria for HD (Table 1). All of these patients were males who resided within the United States of America, and for patients who reported their race, all were white. One patient did not have documentation of an EMG but met all the inclusion criteria and was considered to have HD on the basis of clinical criteria. The mean duration of symptoms at the time of the MR imaging was 6.6 years (range, $0.4-43$ years).

Seventeen patients did not meet the clinical inclusion criteria for HD. These included 11 males and 6 females. Sixteen of these patients resided in the United States, and 1, in Australia. Fourteen patients were white. All except 1 patient had an EMG, but the patient without an EMG had a history that clearly did not support a diagnosis of HD. The diagnoses assigned to the patients without HD were varied. Three patients were diagnosed with motor neuron disease; 2, with amyotrophic lateral sclerosis; 1, with distal muscular dystrophy; 1, with neurogenic thoracic outlet syndrome; 1, with a C8 radiculopathy; 1, with remote poliomyelitis;
1, with an anterior cervical meningeal cyst; 1 , with brachial amyotrophic diplegia; 1, with brachial plexopathy; 1, with a motor neuropathy likely secondary to Sjögren syndrome; 1 , with anterior horn cell disease; 1 , with motor neuron disease versus brachial plexopathy; and 1, with multifocal motor neuropathy. One patient was referred for flexion MR imaging on the recommendation of a radiologist, who noted possible atrophy of the lower cervical spinal cord and loss of cervical lordosis on a prior MR imaging but did not have a final assigned clinical neurologic diagnosis. Therefore, all patients who did not meet the criteria for HD ultimately had either an alternate diagnosis and explanation for symptoms or, in a single case, no neurologic signs or symptoms.

\section{MR Imaging Evaluation}

The diagnostic performance of the studied parameters is listed in Table 2. Four imaging attributes, LOA, dural shift with flexion, consensus diagnosis of neutral images, and consensus diagnosis of combined neutral and flexion images, were all able to discriminate patients with HD from patients without $\mathrm{HD}(P<.05$ for each). The highest diagnostic performance, as measured by the odds ratio, occurred by using the combination of neutral and flexion MR imaging examinations, though it did not differ statistically from other techniques given the limited sample size as assessed by overlapping confidence intervals. Sensitivity was maximized by using the presence of anterior dural shift (76\%; 95\% CI, $55 \%-89 \%)$. Specificity remained high for these 4 techniques with only 1 patient without HD being misclassified on the basis of the LOA and dural shift images, and notably all patients without HD were correctly classified by the readers on both neutral and flexion MR imaging examinations. Although there was little change in sensitivity and specificity with the addition of flexion images, the subjective diagnostic confidence increased. Of the 14 patients classified as having HD by using the neutral images, only 5 were rated with a high confidence level. With the addition of flexion images, 1 of the misclassified patients was determined to have HD, and all 15 patients classified as having HD were rated with a high confidence level. With flexion, the median anterior dural shift in patients with $\mathrm{HD}$ was $3 \mathrm{~mm}$ (mean, $2.7 \mathrm{~mm}$; range, 0-7 $\mathrm{mm}$ ).

Five patients with HD had no anterior dural shift. This subgroup had a mean age of 37.8 years (range, 17-61 years). In the group without $\mathrm{HD}$, only 1 patient had any dural shift $(1 \mathrm{~mm})$, so the odds of having any dural shift were $>50$ times higher in the HD group than in the group without HD $(P<.0001$, Table 2$)$. Figure 1 demonstrates marked MR imaging findings in a patient with $\mathrm{HD}$, and Fig 2 demonstrates more subtle MR imaging findings in a second patient with HD.

The greatest degree of EMG abnormality at the C8 level with severe or very severe involvement (rated 3 or 4 on a 4-point scale) and the presence of LOA were each significantly more likely in patients with HD (Table 2). The difference of spinal cord T2 hyperintensity, asymmetric cord flattening, and abnormal spinal curvature between the 2 groups was not statistically significant.

\section{False-Negative Findings on MR Imaging}

There were 6 patients with a clinical diagnosis of HD that was not detected on MR imaging. These patients were older than those 


\begin{tabular}{|c|c|c|c|c|c|c|c|}
\hline & \multicolumn{2}{|c|}{ Sensitivity } & \multicolumn{2}{|c|}{ Specificity } & \multicolumn{2}{|c|}{ Diagnostic Odds Ratio $^{a}$} & \multirow[b]{2}{*}{$\begin{array}{c}P \\
\text { Value }\end{array}$} \\
\hline & Estimate & $\begin{array}{c}95 \% \\
\text { Confidence } \\
\text { Interval }\end{array}$ & Estimate & $\begin{array}{c}95 \% \\
\text { Confidence } \\
\text { Interval }\end{array}$ & Estimate & $\begin{array}{c}95 \% \\
\text { Confidence } \\
\text { Interval }\end{array}$ & \\
\hline Neutral MRI ${ }^{\mathrm{c}}$ & $70 \%(14 / 20)$ & $(48 \%-85 \%)$ & $100 \%(17 / 17)$ & $(82 \%-100 \%)$ & 78.1 & $(4.0-1505.9)$ & $<.0001$ \\
\hline Neutral and flexion MRI & $71 \%(15 / 21)$ & $(50 \%-86 \%)$ & $100 \%(17 / 17)$ & $(82 \%-100 \%)$ & 83.5 & $(4.3-1605.1)$ & $<.0001$ \\
\hline LOA on neutral images ${ }^{c}$ & $65 \%(13 / 20)$ & $(43 \%-82 \%)$ & $94 \%(16 / 17)$ & $(73 \%-99 \%)$ & 29.7 & $(3.2-273.4)$ & .0004 \\
\hline Anterior dural shift with flexion ${ }^{d}$ & $76 \%(16 / 21)$ & $(55 \%-89 \%)$ & $94 \%(16 / 17)$ & $(73 \%-99 \%)$ & 51.2 & $(5.4-488.7)$ & $<.0001$ \\
\hline Asymmetric cord flattening & $48 \%(10 / 21)$ & $(28 \%-68 \%)$ & $65 \%(11 / 17)$ & $(41 \%-83 \%)$ & 1.7 & $(0.4-6.2)$ & .521 \\
\hline Cord T2 signal & $33 \%(7 / 21)$ & $(17 \%-55 \%)$ & $94 \%(16 / 17)$ & $(73 \%-99 \%)$ & 8.0 & $(0.9-73.2)$ & .053 \\
\hline Abnormal curvature ${ }^{c}$ & $50 \%(10 / 20)$ & $(30 \%-70 \%)$ & $65 \%(11 / 17)$ & $(41 \%-83 \%)$ & 1.8 & $(0.5-6.9)$ & .510 \\
\hline $\begin{array}{l}\text { C8 most affected cervical myotome at } \\
\text { EMG with moderate-to-severe change }\end{array}$ & $50 \%(10 / 20)$ & $(30 \%-70 \%)$ & $88 \%(14 / 16)$ & $(64 \%-97 \%)$ & 7.0 & $(1.3-39.1)$ & .0317 \\
\hline
\end{tabular}

a The odds ratio for neutral MRI and neutral and flexion MRI was computed by adding 0.5 to each cell (ie, the empiric odds ratio) to account for the zero cell that resulted from $100 \%$ specificity.

${ }^{\mathrm{b}} P$ values were derived from a 2-tailed Fisher exact test.

c One patient did not have neutral images for assessment.

${ }^{d}$ Recorded as present, defined as any degree of dural shift, or absent.

e One patient did not have an EMG on record.
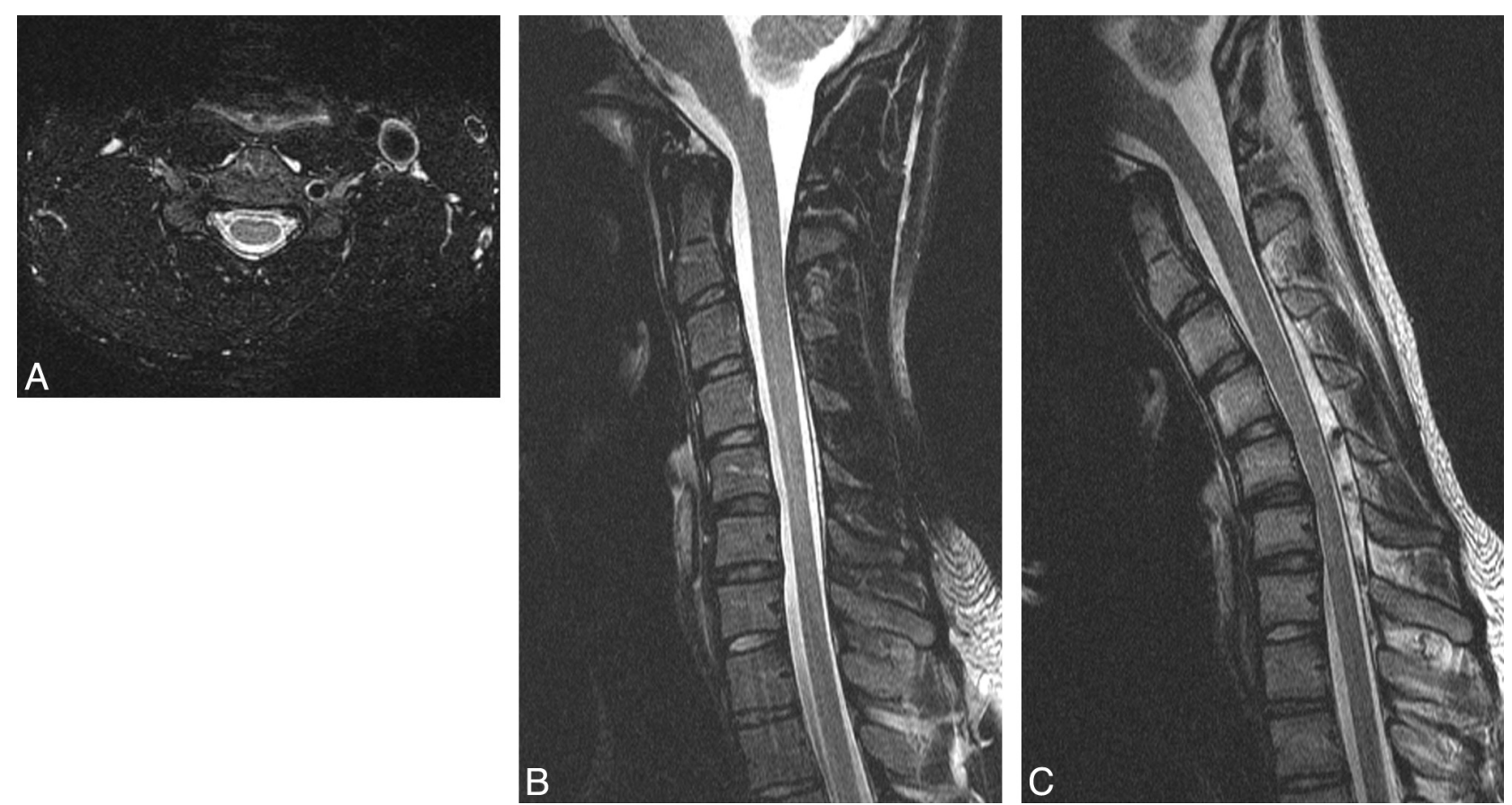

FIG 1. An 18-year-old man with HD. A, Axial T2-weighted image demonstrates LOA at the C5 level. B, Neutral T2-weighted image demonstrates subtle atrophy at C5-C6. C. Flexion T2-weighted image demonstrates $6 \mathrm{~mm}$ of anterior dural shift with near-complete obliteration of the subarachnoid space at C5-C6.

with MR imaging evidence of HD (35.4 years versus 19.7 years, $P=.002)$. LOA was not present in $60 \%$ (3/5, one not assessed due to lack of neutral studies) of these patients. Anterior dural shift with flexion was absent in $67 \%(4 / 6)$ of patients and was only noted as $1 \mathrm{~mm}$ in the remaining 2 patients.

\section{DISCUSSION}

This retrospective study confirms that HD occurs in the North American white population. Early and accurate diagnosis of HD is of considerable prognostic value because HD typically displays a more benign course of clinical stability after a period of progression than alternate diagnoses such as amyotrophic lateral sclerosis or a multifocal motor neuropathy affecting the $\mathrm{C} 8$ or T1 myotomes. We report high MR imaging specificity and moderately high sensitivity in this population, even without consideration of flexion series data. The sensitivity was similar for neutral (70\%) and flexion (71\%) studies. This result indicates that findings of HD, though potentially subtle, are often present on neutral studies, though the diagnostic confidence may be less. Therefore, radiologists should maintain a high level of clinical suspicion for HD, especially in the adolescent and young adult male population referred for MR imaging for asymmetric muscle weakness and atrophy involving the lower cervical and T1 distributions. Careful evaluation of neutral studies for loss of dural attachment, asymmetric cord atrophy, T2 hyperintensity, and abnormal cervical curvature may allow the radiologist both to include a flexion sequence in the MR imaging examination and to suggest the diagnosis of HD. 

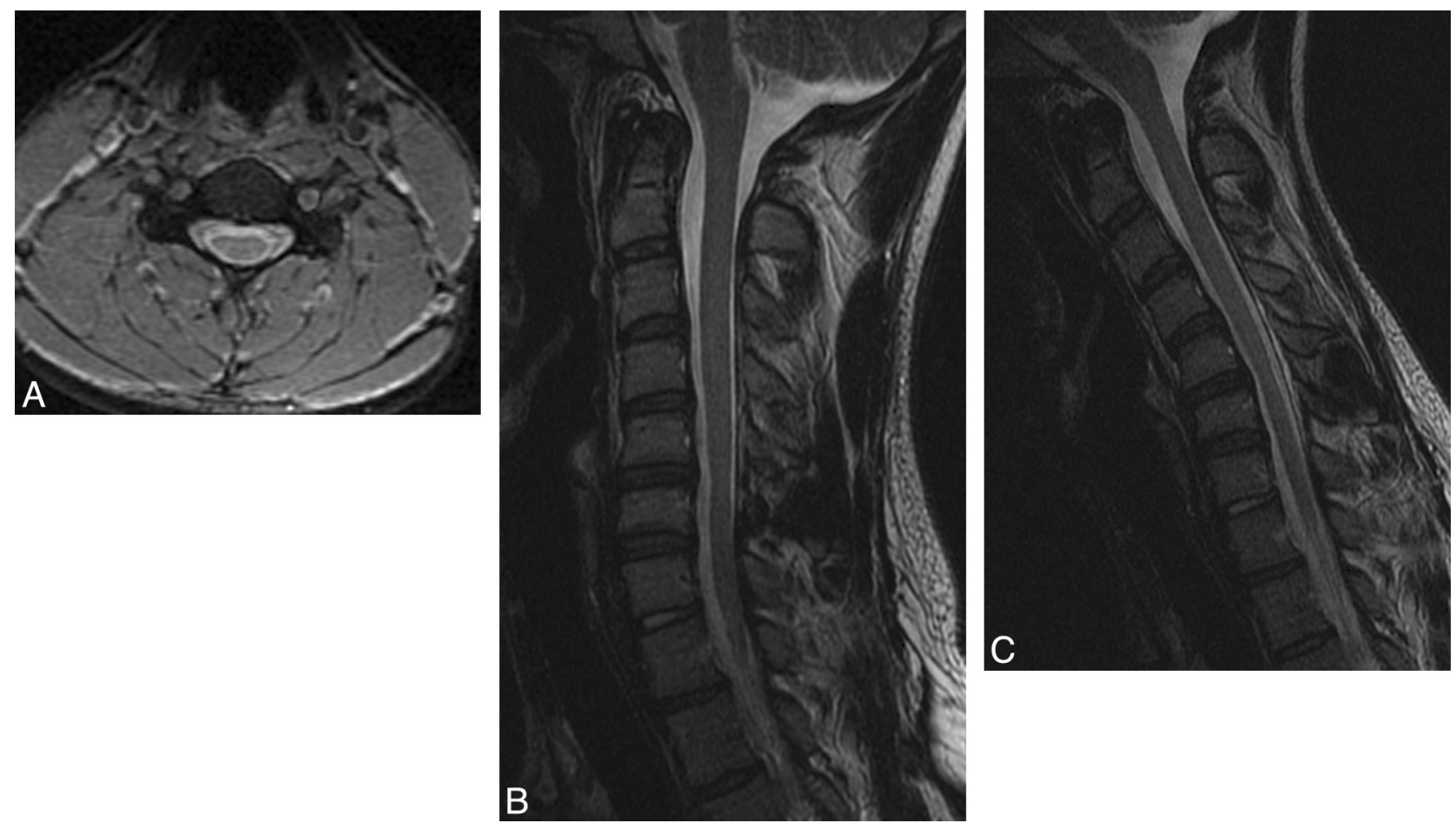

FIG 2. A 20-year-old man with HD. A, Neutral axial gradient-echo image at the C5 level demonstrates subtle bilateral LOA along the lateral aspects of the lamina bilaterally and spinal cord atrophy, asymmetric to the right. $B$, Neutral sagittal T2-weighted image also localizes this atrophy to C5-C6. C, Flexion sagittal T2-weighted image demonstrates $2 \mathrm{~mm}$ of anterior dural shift. The posterior subarachnoid space is not completely obliterated, and there is no direct spinal cord compression.

Most interesting, even when we included flexion data, there was still a $29 \%$ false-negative rate, which included 4 patients with HD with no anterior dural shift with flexion. This is similar to a $32 \%$ false-negative rate previously reported by Tashiro et $\mathrm{al}^{1}$ in a Japanese population. Possible explanations include the older age of these patients at the time of MR imaging (37.8 years), because the findings of HD reportedly decrease with age. ${ }^{2}$ Although the prevailing theory indicates that $\mathrm{HD}$ is a flexion-induced myelopathy resulting from a dura that becomes taut and forwardly displaced in flexion, the underlying pathomechanism is still debated. ${ }^{6}$ We found a wide range of dural shift in patients with HD $(0-7 \mathrm{~mm})$.

The rate of anterior dural shift in our control group was lower than that previously reported by Lai et al, ${ }^{14}$ who observed anterior dural shift with flexion MR imaging in $46 \%$ of 50 healthy individuals in Hong Kong. Possible explanations for this discrepancy include a younger control group (20 years compared with 41 years), other demographic differences between patients in Hong Kong and North America, and a standardized $40^{\circ}$ neck flexion with the aid of a positioning device in their study. Yin et $\mathrm{al}^{15}$ and Hirayama and Tokumaru ${ }^{2}$ both found no anterior dural shift, as defined by a crescent-shaped epidural signal with flexion in 12 healthy controls and 14 healthy controls, respectively. These apparently discrepant findings indicate that further study of the findings on flexion MR imaging of healthy patients is needed. If anterior dural shift is truly present in nearly half of all healthy young males, the significance of this finding in isolation is diminished.

Chen et $\mathrm{al}^{8}$ concluded that LOA is the most accurate finding of
HD on neutral studies, reporting a sensitivity of $93 \%$, though no other follow-up study has confirmed this, to our knowledge. However, we report a lower sensitivity, $70 \%$, indicating that radiologists should not be over-reliant on this single parameter in the North American population. We did, however, find a $100 \% \mathrm{spec}-$ ificity of LOA, similar to the findings by Chen et al. Therefore, LOA is rarely present in healthy North American patients, and the presence of LOA should prompt consideration of HD.

Our study has several limitations. The retrospective design precluded precise standardization of MR imaging protocols, standardization of the degree of neck flexion, and the ability to recruit an age-matched group without HD. For the purposes of this study, we evaluated MR imaging findings at only 1 time point for patients with HD; however, in our experience and that of Hirayama and Tokumaru, ${ }^{2}$ the severity of MR imaging findings can change with time. Although several races were not represented in our study, we believe that the represented races likely reflect the demographic composition of our North American referral population.

\section{CONCLUSIONS}

MR imaging findings in white North American patients with HD include LOA on neutral images and forward displacement of the dura with flexion. Findings are often present on neutral MR images and, in the appropriate clinical scenario, should prompt flexion MR imaging to evaluate anterior dural shift in addition to careful inspection of neutral images for LOA. We report high specificity and moderately high sensitivity of MR imaging for the diagnosis of HD.

AJNR Am J Neuroradiol 34:451-56 Feb 2013 www.ajnr.org 


\section{REFERENCES}

1. Tashiro K, Kikuchi S, Itoyama Y, et al. Nationwide survey of juvenile muscular atrophy of distal upper extremity (Hirayama disease) in Japan. Amyotroph Lateral Scler 2006;7:38-45

2. Hirayama K, Tokumaru Y. Cervical dural sac and spinal cord in juvenile muscular atrophy of distal upper extremity. Neurology 2000;54:1922-26

3. Blumen SC, Drory VE, Sadeh M, et al. Mutational analysis of glycyltRNA synthetase (GARS) gene in Hirayama disease. Amyotroph Lateral Scler 2010;11:237-39

4. Cerami C, Valentino F, Piccoli F, et al. A cervical myelopathy with a Hirayama disease-like phenotype. Neurol Sci 2008;29:451-54

5. Raval M, Kumari R, Dung AA, et al. MRI findings in Hirayama disease. Indian J Radiol Imaging 2010;20:245-49

6. Schröder R, Keller E, Flacke S, et al. MRI findings in Hirayama's disease: flexion-induced cervical myelopathy or intrinsic motor neuron disease? J Neurol 1999;246:1069-74

7. Badve C, Pruthi S. Hirayama disease. Pediatr Radiol 2010;40:1711

8. Chen CJ, Hsu HL, Tseng YC, et al. Hirayama flexion myelopathy: neutral-position MR imaging findings-importance of loss of attachment. Radiology 2004;231:39-44

9. Donofrio PD. AAEM case report \#28: monomelic amyotrophy. Muscle Nerve 1994;17:1129-34

10. Gandhi D, Goyal M, Bourque PR, et al. Case 68: Hirayama disease. Radiology 2004;230:692-96

11. Tynan J, Frangou E, Voll C, et al. Hirayama disease. Can J Neurol Sci 2010;37:703-05

12. Huang YL, Chen CJ. Hirayama disease. Neuroimaging Clin N Am 2011;21:939-50, ix-x

13. Agresti A. Categorical Data Analysis. Hoboken, New Jersey: John Wiley and Sons; 2002

14. Lai V, Wong YC, Poon WL, et al. Forward shifting of posterior dural sac during flexion cervical magnetic resonance imaging in Hirayama disease: an initial study on normal subjects compared to patients with Hirayama disease. Eur J Radiol 2011;80:724-28

15. Yin B, Liu L, Geng DY. Features of Hirayama disease on fully flexed position cervical MRI. J Int Med Res 2011;39:222-28 